\title{
HÔTEL OLD CATARACT: IMAGE, TEXTE
}

Cette photographie, reproduite à la une de Libération le lendemain de la mort d'Hervé Guibert, ${ }^{1}$ se présente d'emblée comme un défi à la parole. Guibert, bouche-bée, contemple son image dans le miroir. Ou plutôt, c'est son reflet dans le miroir qui lui renvoie l'interdiction absente du double nimbé, mais aperçu de dos, de façon que c'est l'homme qui appartient au monde de l'inconnu, de la menace, tandis que le visage du fantôme, tout muet qu'il est, exprime de forts sentiments sans noms. Ainsi le va et vient troublant du corps et de son image commence légèrement à frôler les possibilités du dire.

Depuis ses observations sur l'indicible des photos familiales dans L'Image Fantôme (pp.38 et 66) jusqu'au moment où Le protocole compassionnel renonce à l'écriture du sida en faveur de la vidéo La Pudeur et l'Impudeur, le texte et l'image occupent des positions réciproques mais contradictoires dans l'oeuvre de Guibert. Méditer Hôtel Old Cataract, c'est vite se rendre compte que ce sont les mots qui manquent. Surpris en train de se surprendre, l'auteur cède son image (ses images à proprement dire, puisque, comme cela se passe tellement souvent dans son écriture, c'est le dédoublement de Guibert en fantôme qui occupe et au même moment qui déjoue le studium de ce portrait) à l'exécution dépossédante de la photographie. Déclic double, tout d'abord de l'objectif de Berger qui constitue l'ébranlement dans l'ordre de ce qu'on peut savoir de l'image, des représentations qu'on peut faire du corps_-et plus tard de Guibert lui-même-par l'actionnement du regard photographique porté sur l'ébahissement de sa propre image, qui le constitue en anticipation du tirage définitif.

Assiste-t-on donc à une mise-en-scène recherchée, destinée à suggérer l'autoportrait faux dans le domaine de la photographie comme dans celui des romans fictifs et des autobiographies inventées de Guibert? L'attribution de la séance à Hans Georg Berger ne suffit pas à dissiper le sentiment de malaise que c'est une fois de plus Guibert qui hante sa propre apparition dans la glace. De toute manière, la distinction entre le sujet et le photographe, pour Guibert, ne prend pas les dimensions attendues: comme il l'indique dans le texte qui accompagne la série de photographies de Berger, 'Le photographe et son modèle jouent à la photographie jusqu'au délire." Aucun délire sans composition, peut-être, mais Guibert va plus loin en associant au jeu la décomposition, l'abolition des écarts qui délimitent le corps sien et celui d'autrui. La parole de L'Image de Soi ou L'Injonction de son Beau Moment commence à commenter l'image, et on n'est nullement étonné de découvrir que le commentaire s'efforce de rejeter la transformation simple du photographique en romanesque. Bien que la fascination que Berger lui apporte ait l'effet de transformer son modèle en 'l'acteur de toutes ces fantaisies' (L'Image de Soi), cette obsession, elle aussi, trouve son double dans le narcissisme de Guibert, substantif que l'auteur cherche à réinventer en lui accordant (et voilà que l'image, cette fois-ci, se dissipe en des mots)

1 'Guibert, une mort écrite', Libération, 28-29 décembre 1991, p.1.

2 L'Image de Soi, ou L'Injonction de son Beau Moment?, in Dialogue d' images, Images de Hans Georg Berger (Bordeaux, William Blake and Co., 1988). Le texte est de Guibert, qui est aussi le sujet de chacune des photographies. Les références futures seront citées dans le texte sous le titre: L'Image de Soi. 
toute sa fatalité mythique. Plutôt que 'la lente émaciation' de la décomposition (aussi bien que de la maladie), il souhaite que les images de Berger représentent un corps qui atteigne à la perfection du cadavre-signifiant menteur du premier rang. Comme si on pouvait contempler son propre visage au moment de la mort. Telle est la proposition que le revenant dans la glace fait au Guibert qui l'enfante. Ainsi qu'Hôtel Old Cataract, les écrits de Guibert, d'une façon toujours imprévisible, témoignent pour leur part de l'importance de ce souci en ce qui concerne les relations entretenues avec ses proches et, également, avec lui-même.

Que ce soit l'image manigancée de cette photographie, ou l'aparté façonné du Protocole compassionnel:

C'est comme un miroir, on s'habitue à son propre miroir et quand on se retrouve dans un miroir inconnu à l'hôtel, on voit autre chose. Le regard des autres me fait sentir moi-même une autre personne que celle que je croyais être (pp.120-121),

le refus obstiné de l'identité fixe, facile, s'introduit dans chacun des projets de Guibert comme un projet mille fois recommencé mais jamais achevé. Dans le regard abasourdi du reflet, on devine l'immatérialité du jeu de la lumière, la transformation du corps en image-en même temps que le geste dépassé que fait Berger pour ressaisir ce corps et ce regard au (beau) moment de leur 'évaporation' ( $L$ 'Image de Soi), et, une méconnaissance de soi qui défait tous nos préjugés sur l'identité.

'Maintenant la limite précise de la réalité devient floue, et les mensonges de l'histoire personnelle sont en suspens'. Guibert caractérise ainsi un moment de la suite des changements photographiques de Duane Michals ${ }^{3}$ qu'il commente. Pour Guibert, des termes comme la mort et l'amour ne réussissent à décrire ni nos propres expériences du corps ni nos relations avec les autres. Terroriste dans ses attentats mortels contre la tyrannie du 'je' permissible, ce n'est pas seulement la réinvention du moi qui s'ensuit dans ses écrits 'incroyables mais vrais' ( $L$ 'Image de $S o i$ ), mais aussi l'éloge d'une tendresse inimaginable des perspectives de la légitimité: on pourrait citer les actes infâmes des brigands contre les enfants de Vous $m$ 'avez fait former des fantômes, les liens impertinents entre les deux paires d'amants des Chiens, le sort qui crée des interdépendances néfastes entre Guibert et Bill vers la fin d'A l'ami, Mon valet et moi et ses relations vicieuses ....

Hôtel Old Cataract vient s'insérer dans cette liste. Berger y discerne le 'Beau moment', mais sous injonction, et c'est Guibert qui lui rend sa beauté fragile dans son texte-en le 'dévoilant' à peine, juste assez pour suggérer une relation romanesque entre lui et Berger, entre Guibert et lui-même:

Je suis seul vis-à-vis des photographies de Hans Georg Berger comme vis-à-vis de ma propre écriture: à la fois au plus près d'elle-même, et déjà si lointain.

(L'Image de Soi.)

3 Duane Michals, Changements (Paris: Herscher, 1981), 'Le Texte de Duane Michals a été établi par Hervé Guibert en collaboration avec Philippe Stoeckel pour la traduction, d'après un entretien inédit fait à Paris en novembre 1978, et quelques lettres'. 
Une dizaine de jours avant l'incommunication abrupte et répétée qui deviendrait Hôtel Old Cataract, Guibert écrit d'Egypte à Suzanne, la grand-tante qu'il avait déjà photographiée dans Suzanne et Louise en simulacre de la mort,

au moment de ta mort, (...) ton regard viendra se poser au-dessus du mien, ou en-dessous, comme un verre de contact d'âme à âme. Es-tu déjà morte?"

Le sort thanatologique et érotique commun qui lie Hervé et Suzanne se transmet-il aux spectateurs d'Hôtel Old Cataract à leur tour? Guibert ne tira pas la pseudo-image de Suzanne morte, convaincu que la séquence était surcompliquée par sa mise-en-scène forcée. Cette image rejoint les photographies inachevées des mères de Guibert et de Barthes dont il parle dans L'Image Fantôme, (pp. 11-18, pp.148-49), abolie encore une fois par le texte qui la réinvente en une relation autre, celle qui s'établit entre l'auteur et les lecteurs. D'un mouvement inverse, Hôtel Old Cataract promet des réinterprétations tout aussi accablantes dans le champ de la photographie, taisant d'un cil juste l'indicible de la mort et de l'amour afin de les représenter comme rien moins que les fantômes de nos propres regards. 